\title{
The Recognition and Research of Improved Probabilistic Neural Network
}

\author{
Xiaojing Shang \\ Electrical and Information Engineering Department \\ The City College Of Jilin Architectural And Civil Engineering Institute, Changchun, 130000, China
}

(E-mail: 312511605@qq.com).

\begin{abstract}
Probabilistic neural network compared with the traditional BP neural network structure is simpler and it is faster to be identificated, so it is widely used in the field of pattern recognition. This paper is mainly focused on similar gesture recognition research, propose an probabilistic neural network gesture recognition algorithm. The simulation results show that the improved probabilistic neural network algorithm on the recognition rate and training time is better than the traditional BP network.
\end{abstract}

Keyword: Pattern Recognition; Feature Extraction; Particle Swarm Optimization; Probabilistic Neural Network

\section{FOREWORDS}

Surface EMG (sEMG) [1] is biological signals from the skin surface by the electrode guiding the neuromuscular system activities recorded.

It reflects the function and state of the nerve, muscle. The different gestures corresponding to different muscle contraction mode, the differences between these patterns reflect in sEMG characterized differences.

As an important bio-electrical signal, sEMG is widely used in bionics, bio-feedback, sports medicine and rehabilitation works.

As to hand signal action principles of pattern recognition method, at present, home and abroad has all got some achievements , such as Rencheng Wang and others has made use of BP neural networks to be in the progress to doing a star injustice, stretching the wrist, introversion whorl wrist and extroversion whorl wrist four species actions for instance identification. At 2000, Francis has brought forward one kind of muscle electric signal mixing up the model controlling; its rightness recognition rate can be on a par with manpower neural networks ANN method appearance. At 2004, Kiguchi. $\mathrm{K}$ and others makes use of nerve blurred controller with layers to have studied principles of pattern recognition of the upper limb. These distinguish method basically detachable, be [2] count identification method, neural networks distinguishes method [3-5], [7] grade distinguish [6] , integrated identification of syntax method, neural networks among them distinguish the method sum method is other parallel , have stronger fault-tolerant sex and certainly fit in with learning ability, have been able to distinguish or deformable entering pattern with noise, have got broad usage.

Equality adopt $\mathrm{K}-\mathrm{W}$ to check that method checks the surface muscle electric signal drawing first is worth the main body of a book, method difference, power spectrum density carry out characteristic choice, the strongest characteristic choosing the classification ability is the entering characteristic improving probability neural networks. Middle radial probability neural networks base the function propagation rate has being bound to affect to network function, that the person is worth a sort all is to set up by hand but, repeated modification finds and then right value. The method (PSO) adopt the particle group optimization comes to owe a parameter for sure the main body of a book, can use entire identification process simplification.

The probability neural networks adopt an improvement extends to model " $\mathrm{C}$ " , the forefinger , stretching a wrist, downward, bending a wrist, clenching self's fist, carrying out principles of pattern recognition to inclining bending the wrist, the palm expanding 7 kinds of hand signal actions is the main body of a book study to concentrate on.

\section{SEMG CHARACTERISTIC DRAWS AND THE CHARACTERISTIC CHOOSES}

Choice being hit by a characteristic in principles of pattern recognition is very important, the characteristic is no appropriate or no sufficient if being put into use, may reduce accurate rate distinguishing. The characteristic number is inadvisable to be inadvisable to be short very much very excessively also, the general initial stage distinguishing a scheme in design stage ought to try one's best to list out the various characteristic possibly relevant to classification excessively, such can fully utilize various useful information , improvement classification effect. The characteristic is able to bring difficulty to difficulty calculation but, very much, that more than the correct or required number data need to occupy magnanimous memory space compose in reply calculation time, and be able to bring about classification effect worsening. The characteristic being in progress in two aspects picks up the main body of a book respectively from the time domain and frequency region, adopt K-W checkout method to carry out the most effective characteristic appraising , electing out of classification on single characteristics and then.

\section{A. The feature extraction based on time domain}

Time domain analysis is the basic of electromyography signal analysis method, because of its feature extraction is relatively simple, in multi-channel SEMG processing application is very extensive. This paper chooses mean and variance as the electromyography signal time domain features.

(1) Calculating the mean value

$$
x_{\text {iemg }}=\frac{1}{N} \sum_{i=0}^{N-1}\left|x_{(i)}\right|
$$

Among them $x_{(i)},(i=0,1,2 \ldots, N-1)$ is the length of time series for $\mathrm{N}$. 
(2) Variance computation

$$
V A R=\frac{1}{N-1} \sum_{i=0}^{N-1}\left|x^{2}(i)\right|
$$

\section{B. Based on frequency domain feature extraction}

Physical activities, muscle contraction force size depends on the motor nerve fiber efferent action potential frequency, thus use power spectral density to represent the characteristics of sEmg signals is appropriate. So based on the above theoretical basis and the facts, the power spectrum peak near the shape parameter as the electromyography signal characteristic, namely electromyography signal power spectrum of bandwidth can characterization of sEmg signals represent movement patterns.

In practical application, the power spectrum of the bandwidth of the reciprocal as electromyography signal characteristics, bandwidth with equivalent rectangular bandwidth said. Because of multi-channel semg only in the $100-400 \mathrm{~Hz}$ range, so power spectrum characteristic value calculation for:

$$
Q=\frac{1}{B}=\frac{p\left(f_{0}\right)}{\int_{0}^{400} p(f) d_{f}}
$$

Through the above calculation we get six features the first channel mean, the second channel mean, the first channel variance, the second channel variance, the first channel power spectral density, the second channel power spectral density. With K - W inspection method for each feature for inspection, choose the most powerful classification characteristics.

Calculated through programming various characteristics of the statistical $\mathrm{H}$ value such as table 1 , from the table results can be seen in the time domain features 1 channel mean and 2 channel mean statistical quantity is big, the frequency domain characteristics of 1 channel power spectrum density and 2 channel power spectral density statistical quantity is big, namely the four characteristics of the classification ability is strong, and can from the time-frequency domain two aspects fully embody the signal feature. So, choose the four characteristics as recognition characteristic of sample input.

TABLE I. THE VALUE OF STATISTICS H FOR 6 KINDS OF SURFACE EMG CHARACTERISTICS

\begin{tabular}{|c|c|c|c|}
\hline \multirow{2}{*}{$\begin{array}{c}\text { Valye of H } \\
\text { Channeh }\end{array}$} & Mean & Variance & $\begin{array}{c}\text { Power } \\
\text { spectral } \\
\text { density }\end{array}$ \\
\hline 1 & 47.5542 & 7.8069 & 46.6799 \\
\hline 2 & 46.7255 & 12.7143 & 45.3717 \\
\hline
\end{tabular}

III. PROBABILISTIC NEURAL NETWORK MODELS

Probabilistic neural network is put forward in 1990 by Speech [8] a radial basis function (RBF) network important deformation [9]. It USES multivariate Parzen Windows estimate inhomogeneous probability density function and the Bayesian posterior probability as the output, the training time is short, the structure is stable, has strong nonlinear recognition ability, and not easy to converge to a local minimum point, especially suitable for solving pattern recognition problems.

Probabilistic neural network (generally divided into four layers: input layer, model layer, summation layer and output layer. Input layer as the training sample, $\mathrm{X}=(\mathrm{x} 1, \mathrm{x} 2, \cdots, \mathrm{xq}) \mathrm{T}$ to remember, the neuron number and sample vector dimension equal.

In the MATLAB programming a probabilistic neural network to create the code for net = newpnn(P,T,SPREAD), in which represents the network input sample volume and the $\mathrm{T}$ said network target vector, and SPREAD said radial basis function transmission rate.

Aiming at this problem, this paper presents an optimum transmission rate means that the particle swarm optimization method. In this method, we can determine the optimal transmission rate and simplify the process of identification.

\section{MOdified Probabilistic NeURAL NETWORK IDENTIFICATION}

Particle swarm optimization algorithm (PSO) is a stochastic optimization technique based on the species. It be made by Eberhart and Kennedy in 1995[10]. PSO is a randomized, parallel optimization algorithm. There are many advantages about it. It doesn't require the optimized function is differentiable, derivative, continuous nature, the convergence speed is fast, and the algorithm is simple, easy to program. This paper uses the method of radial basis function to optimize transmission rate. PSO algorithm described below:

This paper is the use of particle swarm optimization algorithm to find a maximize the objective function of the optimal radial basis function transmission rate, $\eta$ represents the target function:

$$
\eta=\frac{\text { The number of correctly classified samples }}{\text { The total number of test samples }}
$$

Improved probabilistic neural network recognition process, such as is shown in Algorithm 2.

Algorithm 2:

Step1: owned by one of the training sample and the weight vector

Step2: initialize the objective function and the control parameter of the PSO algorithm randomly generated contemporary parameter vector XI $(\mathrm{i}=1,2 \ldots \mathrm{N})$, and $\mathrm{xi}$

Step3: normalized samples training PNN, for each particle, the objective function value for comparison with the objective function value of the current local optimal pests profile pests is, the objective function value is larger

Step4: each particle, its objective function value of the objective function value of the current global optimum best compare update best objective function value larger

Step4: According to the formula (9) updates the values of $\mathrm{xi}$ and VI

Step5: termination of discrimination: If the number of iterations is the maximum number of times or the objective function reaches optimal if the loop is terminated, otherwise it returns Step3 
Step6: Back particle swarm global optimum gbest as the optimal value of the propagation rate. The experimental results obtained in this optimal value as the final recognition result

\section{SIMULATION AND RESULTS ANALYSIS}

The improvement of this article probabilistic neural network algorithm denoted as S1 algorithm, and traditional BP neural network algorithm denoted S2 algorithm.

For S1 algorithm11-12]: First determine PNN network structure, according to the characteristics of the radial basis function, and the input layer neurons number of the input sample vector dimension equal to the output layer neuron number is equal to the number of training sample data types, and the output layer of the network is competitive layer, each neuron corresponding to one data category. PNN network structure is designed for: the input layer has four neurons, and the output layers have 7 neural.

Middle layer transfer function for gaussian function, the output layer transfer function is linear function. The mean of 1 channel, the mean of 2 channel mean, first channel power spectral density, the second channel power spectral density as input characteristic, carries on the simulation results as shown in figure 1, figure 2 shows. Mentioned the $\mathrm{K}$ - W inspection methods for feature extraction, and put the best features of classification ability as network input characteristics to improve recognition rate. This paper have carried contrast test, increase the number of features will be the first channel variance as input characteristic, the simulation results as shown in figure 3 shows.

For S2 algorithm [13-14]: first determine the structure of BP network, which includes input layer, hidden layer and output layer. Input layer neuron number and input feature vector dimension is same, output layer neuron number and to identify the types of the action of the same number, and the number of hidden layer neurons by [15] of the formula:

$$
S=\sqrt{m+n}+a
$$

$\mathrm{M}, \mathrm{n}$ were presented input layer and output layer neuron number, for constant, numerical in $1 \sim 10$, it's numerical according to the experimental effect sure.

The design of BP network structure is: input layer has four neurons, hidden eight neurons, output layer 7 neurons, also will be the first channel mean, the second channel mean, first channel power spectral density, the second channel power spectral density as input characteristic, carries on the simulation results as shown in figure 4, shows, the recognition rate of two kinds of methods such as shown in table 2 .

Contrast The figure 2 with figure 3, we choose the typical classification ability characteristics as network input features can reduce error namely improve recognition rate. Based on the experimental data, all kinds of don't exist among part intersection, this is also one of the reasons for the errors. Therefore, the choice of appropriate representative characteristics to improve recognition rate, namely reduce error is very important.
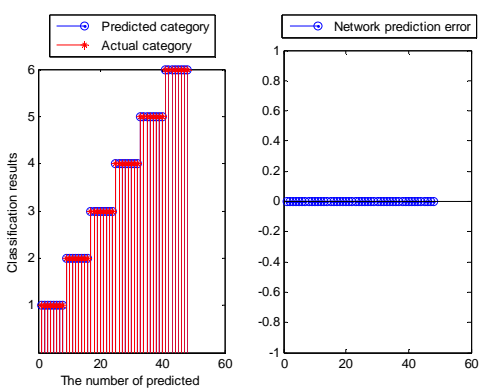

Figure 1 . The effects and error after network training

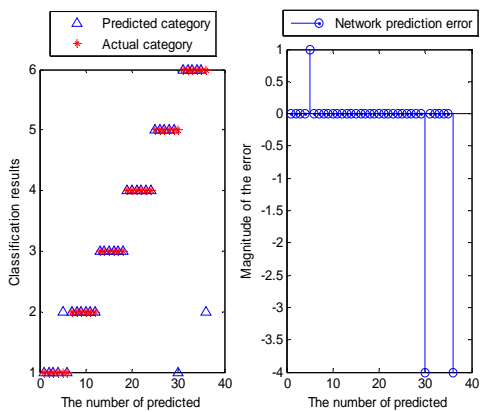

Figure 2. The prediction effect and error of the network
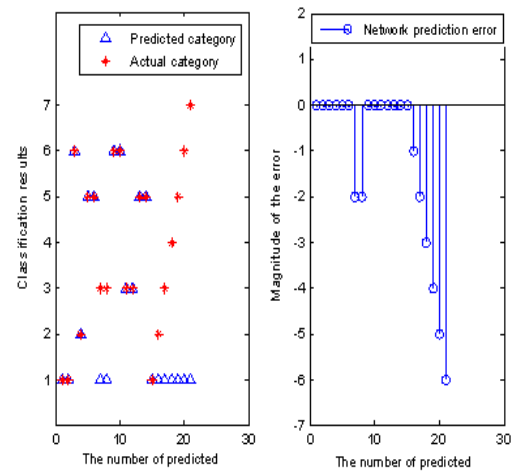

Figure 3. The prediction effect and error of the added characteristic number network

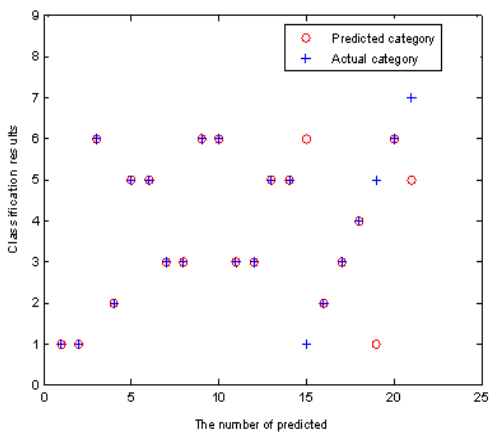

Figure 4. The simulation results of actual class and test class 
TABLE II. RECOGNITION RATE COMPARISON FOR S1 AND S2 ALGORITHM

\begin{tabular}{|c|c|c|}
\hline The algorithm & $\begin{array}{c}\text { The average } \\
\text { recognition } \\
\text { rate }\end{array}$ & $\begin{array}{c}\text { Recognition } \\
\text { time }\end{array}$ \\
\hline S1 & $91 \%$ & 0.38 \\
\hline S2 & $85.7 \%$ & 0.72 \\
\hline
\end{tabular}

From the experimental results, it can be seen that the proposed improved probabilistic neural network algorithm and the traditional BP neural network algorithm, the recognition rate in significant increase, and the training time and shorten the nearly half.

Improved PNN and the traditional BP network compared, in the following aspects has the obvious advantage:

(1) Process is simple, the convergence speed. Improved PNN without the traditional BP neural network error back propagation process, training speed.

(2) The network convergence, high stability. Traditional BP neural network, the connection of each layer of the weights of the training on initial value sensitivity, the classification of the traditional BP neural network, the result is not sure, and BP network classification rule is not sure to explain, lack of transparency.

(3) The sample additional ability is strong, and can tolerate individual wrong sample.

\section{CONCLUSION}

In this paper by using particle swarm optimization algorithm to determine the transmission rate, particle swarm optimization with the probabilistic neural network for seven kinds of hand gestures action for pattern recognition.

Through the simulation experiment proves that this recognition method than the traditional $\mathrm{BP}$ neural network recognition algorithm has a higher identification accuracy, training time also greatly speed up, and easy to realize, there is no getting into the local advantages, have certain generalization ability. Shows that the method is effective.

But, improved probabilistic neural network recognition algorithm there is a need to further improve the recognition rate. Therefore, the next step research emphasis is also looking for more effective recognition algorithm, improve the gestures action identification accuracy.

\section{REFERENCES}

[1] JIN D W, WANG R CH. Artificial intelligent prosthesis[J]. Chinese J. Clinical Rehabilitation, 2002, 5 (20): 2994-2995

[2] Paclik P, Novovicova J, Pudil P. Road sign classification using Laplace classifier [J]. Pattern Recognition Letters, 2000, 21(13 14): $1165 \sim 1173$.

[3] Escalera A de la, Armingol J M,Salchs M A,et al. Road traffic sign detection and classification[J]. IEEE Transactions on Industronics, 1997,44(6): 848 859.

[4] Luo C R, Potlapalli H, Hislop D. Traffic sign recognition in outdoor environments using reconfigurable neural networks [A]. Proceedings of the International Joint Conference on Neural Networks[C]. Nagoya, Japan, 1993:1306 1309.

[5] Estable S, Schick J, Stein F, et al. Real-time traffic sign recognition system [A]. Proceedings of the Intelligent Vehicle' 94 Symposium [C]. Paris, France, 1994:213 218.

[6] Bessere B, Estable S, Ulmer B,et al. Shape classification for traffic sign recognition [A]. Proceedings lst IFAC International Workshop Intelligent Autonomous Vehicles[C].1993:487 492.

[7] Sueteus P, Fua P, Hanson A J. Computational strategies for object recognition[J].ACM Computing Surveys, 1992,24(1): 5 61.

[8] Specht D. Probabilistic neural network[J]. Neural Networks, 1990,3(1):109 118.

[9] McDuff R J, Simpson P K. An Investigation of Neural Networks for F-16 Fault Dignosis:II. System Performance[A].SPIE 1294 Application of Artificial Neural Networks[C]. 1990:42 45.

[10] Kennedy J, Eberhart R C, Shi Y. Swarm Intelligence. San Francisco, USA: Morgan Kaufmann,2001.

[11] FeiSi Science and Technology Research Center. Neural Network Theory and Realization in Matlab 7[M].Beijing: Publishing House of Electronics Industry, 2005.

[12] LEI Zheng-wei, XU Zhang-sui. Fault Diagnosis Method by Fault Importance Based On Probabilistic Neural Networks [J]. Computer Measurement \& Control,2005, 12(2): 107-109.

[13] YU Qing, YANG Jihai, CHEN Xiang, ZHANG Xu. Pattern Recognition of Gestures Surface- Myoelectrogram Signal based on BP Neural Networks. Journal of Biomedical Engineering Research, 2009,28 (1) : 06-10.

[14] XU Dong, WU Zheng. The System Analysis and Design Based On MATLAB 6.x[M].Xi'an:Xi' an Electronic Science and Technology University Press,2002.

[15] CHU Hui, LAI Hui-cheng. An Improved Back - propagation NN Algorithm And Its Appliction[J]. Computer Simulation, 2007, 24 (4) : $75-77$. 